\title{
Generalized Fuzzy Torus and its Modular Properties ${ }^{\star}$
}

\author{
Paul SCHREIVOGL and Harold STEINACKER
}

Faculty of Physics, University of Vienna, Boltzmanngasse 5, A-1090 Vienna, Austria

E-mail: paul.schreivogl@univie.ac.at, harold.steinacker@univie.ac.at

Received June 19, 2013, in final form October 11, 2013; Published online October 17, 2013

http://dx.doi.org/10.3842/SIGMA.2013.060

\begin{abstract}
We consider a generalization of the basic fuzzy torus to a fuzzy torus with non-trivial modular parameter, based on a finite matrix algebra. We discuss the modular properties of this fuzzy torus, and compute the matrix Laplacian for a scalar field. In the semi-classical limit, the generalized fuzzy torus can be used to approximate a generic commutative torus represented by two generic vectors in the complex plane, with generic modular parameter $\tau$. The effective classical geometry and the spectrum of the Laplacian are correctly reproduced in the limit. The spectrum of a matrix Dirac operator is also computed.
\end{abstract}

Key words: fuzzy spaces; noncommutative geometry; matrix models

2010 Mathematics Subject Classification: 81R60; 81T75; 81T30

\section{Introduction}

In recent years, matrix models of Yang-Mills type have become a promising tool to address fundamental questions such as the unification of interactions and gravity in physics. Their fundamental degrees of freedom are given by a set of operators or matrices $X^{A}$ acting on a finiteor infinite-dimensional Hilbert space. Specific Yang-Mills matrix models appear naturally in string theory [5, 13], and provide a description of branes, as well as strings stretching between the branes.

It is well-known how to realize certain basic compact branes in the framework of matrix models. For example, the noncommutative torus $T_{\theta}^{2}$ as introduced by Connes [7] arises in certain types matrix model compactifications, via generalized periodic boundary condition. A rich mathematical structure has been elaborated including e.g. U-duality and Morita equivalence of the projective modules $[7,10,11]$, which is related to T-duality in string theory. However, these results arise only due to the infinite-dimensional algebra of the non-commutative torus $T_{\theta}^{2}$, which includes a non-trivial "winding sector" of string theory.

In contrast, we will focus in this paper on the class of fuzzy spaces given by the quantization of symplectic spaces with finite symplectic volume. They arise in matrix models not via compactification of but rather as embedded sub-manifolds, or "branes". Their quantized algebra of functions is given by a finite-dimensional simple matrix algebra $\mathcal{A}_{N}=M_{N}(\mathbb{C})$, without any additional sector. As a consequence, concepts such as Morita equivalence do not make sense a priori, and the geometry arises in a different way. A simple and well-known example is the (rectangular) fuzzy torus $T_{N}^{2}$, realized in terms of finite-dimensional clock- and shift matrices. Due to the intrinsic UV cutoff, the fuzzy tori are excellent candidates for fuzzy extra dimensions, along the lines of [4]. The relation between $T_{N}^{2}$ and $T_{\theta}^{2}$ was discussed in detail in [15].

As quantized symplectic manifolds, the noncommutative tori have a priori no metric structure. The infinite-dimensional noncommutative torus $T_{\theta}^{2}$ can be equipped with a differentiable

\footnotetext{
${ }^{\star}$ This paper is a contribution to the Special Issue on Deformations of Space-Time and its Symmetries. The
} full collection is available at http://www.emis.de/journals/SIGMA/space-time.html 
calculus given by outer derivations, and subsequently a metric structure can be introduced via a Laplace or Dirac operator. In contrast, the fuzzy torus $T_{N}^{2}$ admits only inner derivations. However if realized as brane in matrix models, it inherits an effective metric as discussed in general in $[19,20]$, which is encoded in a matrix Laplace operator. This can be used to study aspects of field theory on $T_{N}^{2}[6]$, along the lines of the extensive literature on other fuzzy spaces such as $[1,3,8,14,16]$.

In this work, we study in detail the most general fuzzy torus embedded in the matrix model as first considered in [12], and study in detail its effective geometry. We demonstrate that the embedding provides a fuzzy analogue for a general torus with non-trivial modular parameter. It turns out that non-trivial tori are obtained only if certain divisibility conditions for relevant integers hold, in particular $N$ should not be prime. In the limit of large matrices, our construction allows to approximate any generic classical torus with generic modular parameter $\tau$. Moreover, we obtain a finite analogue of modular invariance, with modular group $\operatorname{SL}\left(2, \mathbb{Z}_{N}\right)$. The effective Riemannian and complex structure are determined using the general results in [19]. In addition we determine the spectrum of the associated Laplace operator, and verify that the spectral geometry is consistent with the effective geometry as determined before.

The origin for the non-trivial geometries of tori is somewhat surprising, since the embedding in the matrix model is in a sense always rectangular. A non-rectangular effective geometry arises due to different winding numbers along the two cycles in the apparent embedding. This finite winding feature leads to a non-trivial modular parameter and effective metric, due to the non-commutative nature of the branes.

This paper is organized as follows. We first review the classical results on the flat torus, as well as the quantization of the basic rectangular fuzzy torus in the matrix model. We then give the construction of the general fuzzy torus embedding, and determine its effective geometry. Its modular properties are studied, and the modular group $\operatorname{SL}\left(2, \mathbb{Z}_{N}\right)$ is identified. We also compute the spectrum of the corresponding Laplace operator, and determine its first Brillouin zone. Finally we also discuss the matrix Dirac operator in the rectangular case and obtain its spectrum.

\section{The classical torus}

Before discussing the fuzzy torus, we review in detail the geometric structure of the classical torus.

The most general flat 2-dimensional torus can be considered as a parallelogram in the complex plane $\mathbb{C}$, with opposite edges identified. The torus naturally inherits the metric and the complex structure of the complex plane. The shape of the parallelogram is given by two complex numbers $\omega_{1}$ and $\omega_{2}$, as illustrated in Fig. 1. One can think of the vectors $\omega_{1}$ and $\omega_{2}$ as generators of a lattice in the complex plane $\mathbb{C}$. Denoting this lattice by

$$
L\left(\omega_{1}, \omega_{2}\right)=\left\{n \omega_{1}+m \omega_{2}, n, m \in \mathbb{Z}\right\}
$$

a point $z$ on the torus is given by

$$
z=\sigma_{1} \omega_{1}+\sigma_{2} \omega_{2} \simeq \sigma_{1} \omega_{1}+\sigma_{2} \omega_{2}+2 \pi L\left(\omega_{1}, \omega_{2}\right),
$$

with coordinates $\sigma_{1}, \sigma_{2} \in[0,2 \pi]$. These points are identified according to the lattice $L\left(\omega_{1}, \omega_{2}\right)$. Such coordinates $\sigma_{1}, \sigma_{2}$ with periodicity $2 \pi$ will be called standard coordinates. In these standard coordinates, the line element is

$$
\mathrm{d} s^{2}=\frac{1}{2}(d z d \bar{z}+\mathrm{d} \bar{z} d z)=\omega_{1} \bar{\omega}_{1} \mathrm{~d} \sigma_{1}^{2}+\left(\omega_{1} \bar{\omega}_{2}+\omega_{2} \bar{\omega}_{1}\right) \mathrm{d} \sigma_{1} \mathrm{~d} \sigma_{2}+\omega_{2} \bar{\omega}_{2} \mathrm{~d} \sigma_{2}^{2}=g_{a b} \mathrm{~d} \sigma_{1} \mathrm{~d} \sigma_{2} .
$$




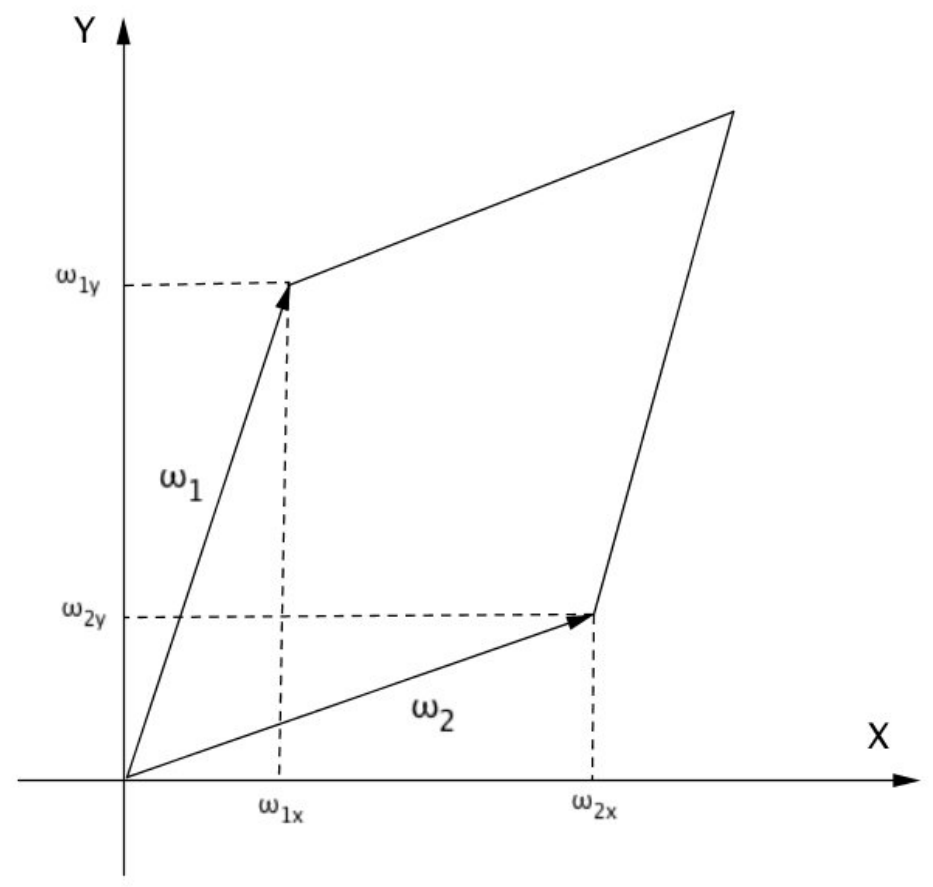

Figure 1. A torus represented as a parallelogram in the complex plane.

We can read off the metric components

$$
g_{a b}=\left(\begin{array}{cc}
\left|\omega_{1}\right|^{2} & \operatorname{Re}\left(\omega_{1}\right) \operatorname{Re}\left(\omega_{2}\right)+\operatorname{Im}\left(\omega_{1}\right) \operatorname{Im}\left(\omega_{2}\right) \\
\operatorname{Re}\left(\omega_{1}\right) \operatorname{Re}\left(\omega_{2}\right)+\operatorname{Im}\left(\omega_{1}\right) \operatorname{Im}\left(\omega_{2}\right) & \left|\omega_{2}\right|^{2}
\end{array}\right) .
$$

Furthermore, we introduce the modular parameter

$$
\tau=\omega_{1} / \omega_{2} \in \mathbb{H},
$$

where $\mathbb{H}$ is the complex upper half-plane $\mathbb{H}=\{z \in \mathbb{C} \mid z>0\}$. We identify conformally related metrics on the torus. Using a Weyl scaling $g \rightarrow e^{\phi} g$ of the metric as well as a diffeomorphism (a rotation), the lattice vectors of the torus can be brought in the standard form $\omega_{1}=\tau$ and $\omega_{2}=1$, see Fig. 2. Then $z=\sigma_{1}+\tau \sigma_{2}$ for $\left(\sigma_{1}, \sigma_{2}\right) \simeq\left(\sigma_{1}, \sigma_{2}\right)+2 \pi(n, m)$. The line element in these standard coordinates then simplifies as

$$
\mathrm{d} s^{2}=\left|\mathrm{d} \sigma_{1}+\tau \mathrm{d} \sigma_{2}\right|^{2},
$$

with metric components

$$
g_{a b}=\left(\begin{array}{cc}
1 & \tau_{1} \\
\tau_{1} & |\tau|^{2}
\end{array}\right)
$$

In these coordinates $z=\sigma_{1}+\tau \sigma_{2}$, one can express the modular parameter through the metric components (2) as follows

$$
\tau=\frac{g_{12}+i \sqrt{g}}{g_{11}}
$$

where $g=\operatorname{det}\left(g_{a b}\right)$. Now on any oriented two-dimensional Riemann surface, there is a covariantly constant antisymmetric tensor ${ }^{1} \frac{1}{\sqrt{g}} \epsilon^{a b}$ with $\epsilon^{12}=-1$. Together with the metric and the

\footnotetext{
${ }^{1}$ This corresponds to the inverse of the volume form.
} 


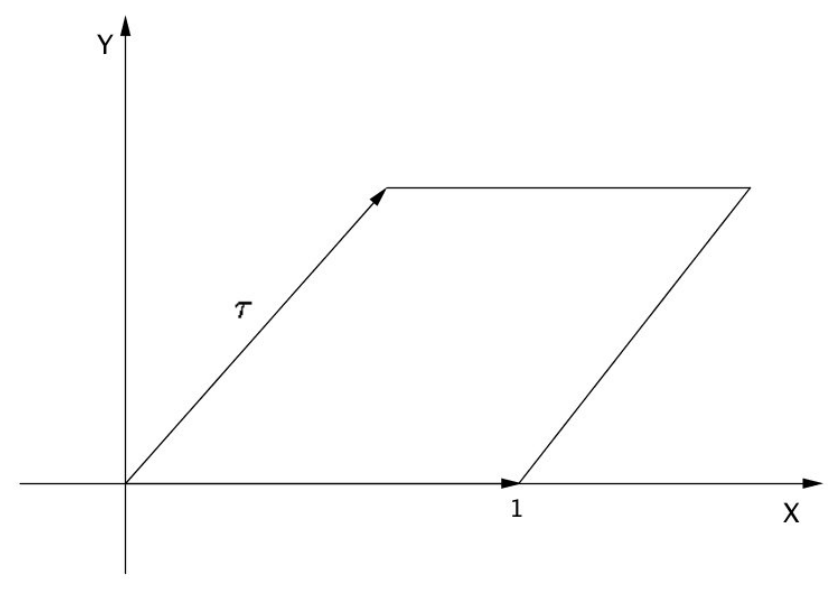

Figure 2. A torus with modular parameter $\tau$.

antisymmetric tensor, we can build the tensor

$$
J_{b}^{a}=\frac{1}{\sqrt{g}} g_{b c} \epsilon^{a c} .
$$

In the above standard coordinates, this tensor is explicitly

$$
J_{b}^{a}=\frac{1}{\tau_{2}^{2}}\left(\begin{array}{cc}
\tau_{1} & -1 \\
|\tau|^{2} & -\tau_{1}
\end{array}\right)
$$

and the square of $J$ is $J^{2}=-1$. It is therefore an almost complex structure. In fact it is a complex structure, since it is constant and thus trivially integrable.

It is instructive to choose Euclidian coordinates $z=x+i y$ on the same torus, with metric $\mathrm{d} s^{2}=\mathrm{d} x^{2}+\mathrm{d} y^{2}$. Then the periodicity becomes $z \simeq z+2 \pi(m+\tau n)$. In these coordinates, the almost complex structure takes the standard form

$$
J_{b}^{a}=\delta_{b c} \epsilon^{a c},
$$

which is

$$
J=\left(\begin{array}{cc}
0 & -1 \\
1 & 0
\end{array}\right)
$$

Now $J^{2}=-1$ is obvious.

Now we can discuss modular invariance. Note that two tori are always diffeomorphic as real manifolds, but not necessarily biholomorphic as complex manifolds. This can be illustrated e.g. with two tori $T_{1}$ and $T_{2}$ defined by the lattice $L\left(\omega_{1}, \omega_{2}\right)=((1,0),(0,1))$ and $L\left(u_{1}, u_{2}\right)=$ $((1,0),(0,2))$, see Fig. 3 . On $T_{1}$ we choose coordinates $\left(x_{1}, y_{1}\right)$, and on $T_{2}$ we choose coordinates $\left(x_{2}, y_{2}\right)$. There is a diffeomorphism

$$
\left(x_{2}, y_{2}\right)=\left(x_{1}, 2 y_{1}\right) .
$$

Let us introduce complex coordinates on tori $z=x_{1}+i y_{1}$ and $w=x_{2}+i y_{2}$. Using the above diffeomorphism, we obtain $w=x_{1}+2 i y_{1}$, and together with

$$
x_{1}=\frac{z+\bar{z}}{2}, \quad y_{1}=\frac{z-\bar{z}}{2 i}
$$



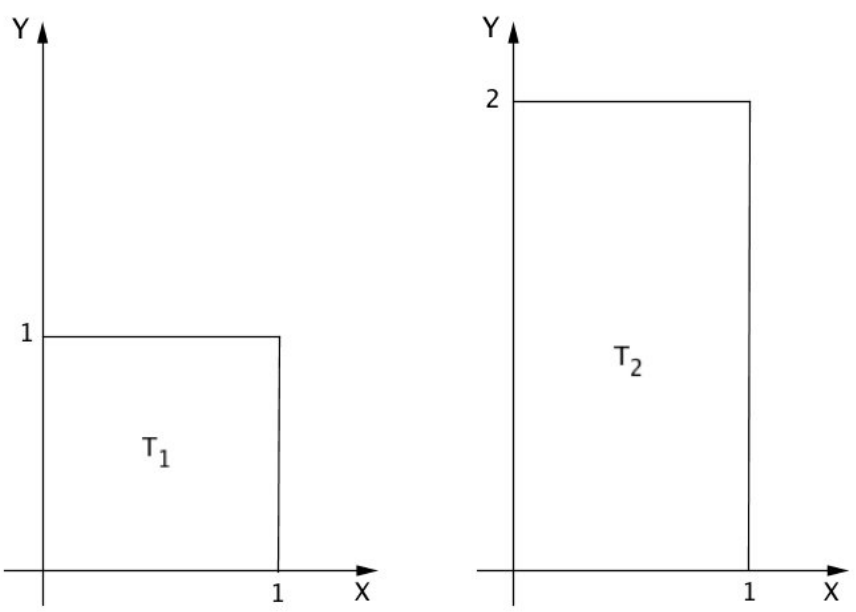

Figure 3. Torus $T_{1}$ and $T_{2}$.

we find

$$
w=\frac{3 z-\bar{z}}{2} .
$$

This is clearly not a holomorphic function of $z$.

Clearly two tori are equal as complex manifolds if their modular parameters $\tau_{\omega}=\omega_{1} / \omega_{2}$ and $\tau_{u}=u_{1} / u_{2}$ coincide. Moreover, two tori are also equivalent if they are related by a modular transformation

$$
\left(\begin{array}{ll}
a & b \\
c & d
\end{array}\right) \in \operatorname{PSL}(2, \mathbb{Z})
$$

To see this, it suffices to note that the two lattices $L\left(\omega_{1}, \omega_{2}\right)$ and $L\left(u_{1}, u_{2}\right)$ are equivalent if they are related by a $\operatorname{PSL}(2, \mathbb{Z})$ transformation

$$
\left(\begin{array}{l}
\omega_{1} \\
\omega_{2}
\end{array}\right)=\left(\begin{array}{ll}
a & b \\
c & d
\end{array}\right)\left(\begin{array}{l}
u_{1} \\
u_{2}
\end{array}\right) .
$$

This leads to fractional transformation of their modular parameters

$$
\tau_{\omega}=\frac{a \tau_{u}+b}{c \tau_{u}+d}
$$

This modular group is in fact generated by two generators

$$
T: \tau \rightarrow \tau+1, \quad S: \tau \rightarrow-1 / \tau,
$$

which obey the relations $S^{2}=(S T)^{3}=1$. The moduli space of $\tau$ is the fundamental domain $\mathcal{F}$, which is the complex upper half-plane $\mathbb{H}$ modulo the projective special linear group $\operatorname{PSL}(2, \mathbb{Z})=$ $\mathrm{SL}(2, \mathbb{Z}) / \mathbb{Z}_{2}$

$$
\tau \in \mathbb{H} / \operatorname{PSL}(2, \mathbb{Z})=\mathcal{F} .
$$

A standard choice for this fundamental domain is $-1 / 2 \leq \tau_{1} \leq 1 / 2$ and $1 \leq|\tau|$, see Fig. 4 . The fundamental domain is topologically equal to the complex plane $\mathcal{F} \simeq \mathbb{C}$. Adding the point $\tau=i \infty$ we obtain the compactified moduli space, which is topological equivalent to the Riemann sphere. The action of the modular transformations $T: \tau \rightarrow \tau+1$ and $S: \tau \rightarrow-1 / \tau$ on the torus is illustrated in Fig. 5. 


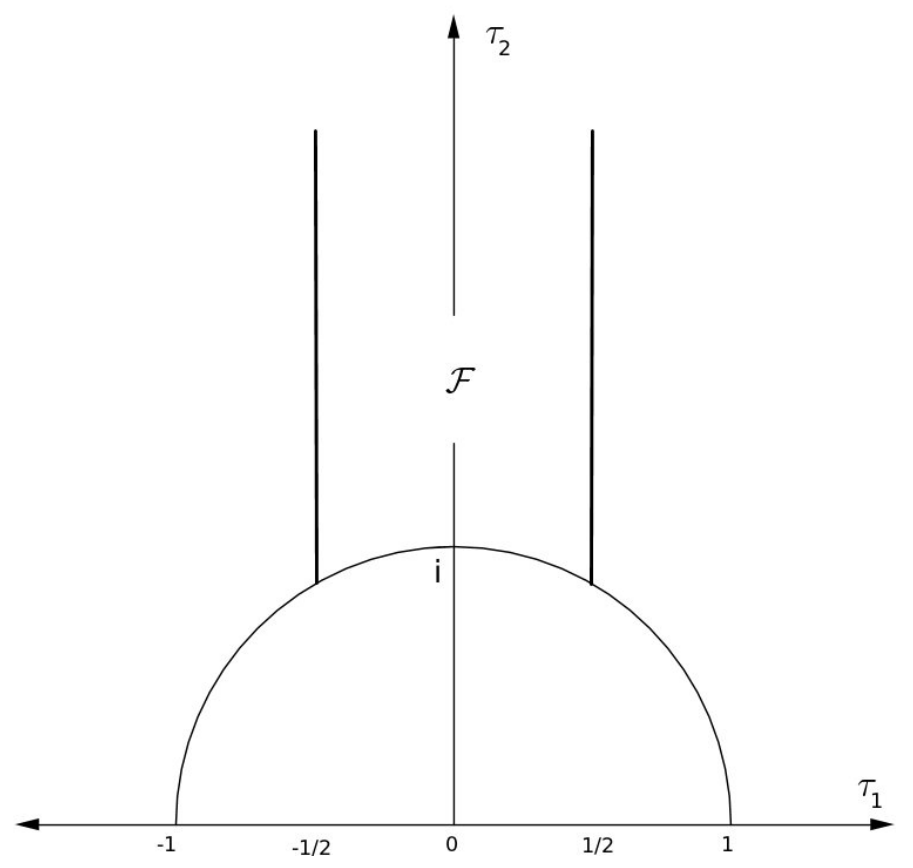

Figure 4. The infinite strip denoted by $\mathcal{F}$ is the quotient space $\mathbb{H} / \operatorname{PSL}(2, \mathbb{Z})$ on the upper half-plane.

\section{Poisson manifolds and quantization}

A Poisson manifold $\mathcal{M}$ is a manifold together with an antisymmetric bracket $\{\cdot, \cdot\}: \mathcal{C}(\mathcal{M}) \times$ $\mathcal{C}(\mathcal{M}) \rightarrow \mathcal{C}(\mathcal{M})$, where $\mathcal{C}(\mathcal{M})$ denotes the space of smooth functions on $\mathcal{M}$. The bracket respects the Leibniz rule $\{f g, h\}=f\{g, h\}+g\{f, h\}$ and the Jacobi-identity $\{f,\{g, h\}\}+$ cyl. $=$ 0 , for $f, g, h \in \mathcal{C}(\mathcal{M})$. The Poisson tensor of coordinate functions is denoted as $\theta^{a b}(x)=$ $\left\{x^{a}, x^{b}\right\}$. If $\theta^{a b}(x)$ is non-degenerate, we can introduce a symplectic form $\omega=\frac{1}{2} \theta_{a b}^{-1} \mathrm{~d} x^{a} \mathrm{~d} x^{b}$ in local coordinates. The dimension of the symplectic manifold $\mathcal{M}$ is always even. The symplectic form is closed $\mathrm{d} \omega=0$, which is just the Jacobi identity. Let us define a quantization map $\mathcal{Q}$, which is an isomorphism of two vector spaces. It maps the space of function to a space of operators

$$
\begin{aligned}
\mathcal{Q}: \quad & \mathcal{C}(\mathcal{M}) \rightarrow \mathcal{A} \subset \operatorname{Mat}(\infty, \mathbb{C}), \\
& f(x) \rightarrow F .
\end{aligned}
$$

In the present context the space of operators will be the simple matrix algebra $\mathcal{A}_{N}=M_{N}(\mathbb{C})$. The quantization map $\mathcal{Q}$ depends on the Poisson structure, and should satisfy the conditions

$$
\mathcal{Q}(f g)-\mathcal{Q}(f) \mathcal{Q}(g) \rightarrow 0, \quad \frac{1}{\theta}(\mathcal{Q}(i\{f, g\})-[\mathcal{Q}(f), \mathcal{Q}(g)]) \rightarrow 0
$$

for $\theta \rightarrow 0$. The algebra $\mathcal{A}$ is interpreted as quantized algebra of functions $\mathcal{C}(\mathcal{M})$ on $\mathcal{M}$. The quantization map $\mathcal{Q}$ is not unique, since higher order terms in $\theta$ are not unique. The natural integration on symplectic manifolds

$$
I(f)=\int \frac{\omega^{n}}{n !} f
$$

is related to its operator version

$$
\mathcal{I}(F)=(2 \pi)^{n} \operatorname{Tr} F
$$



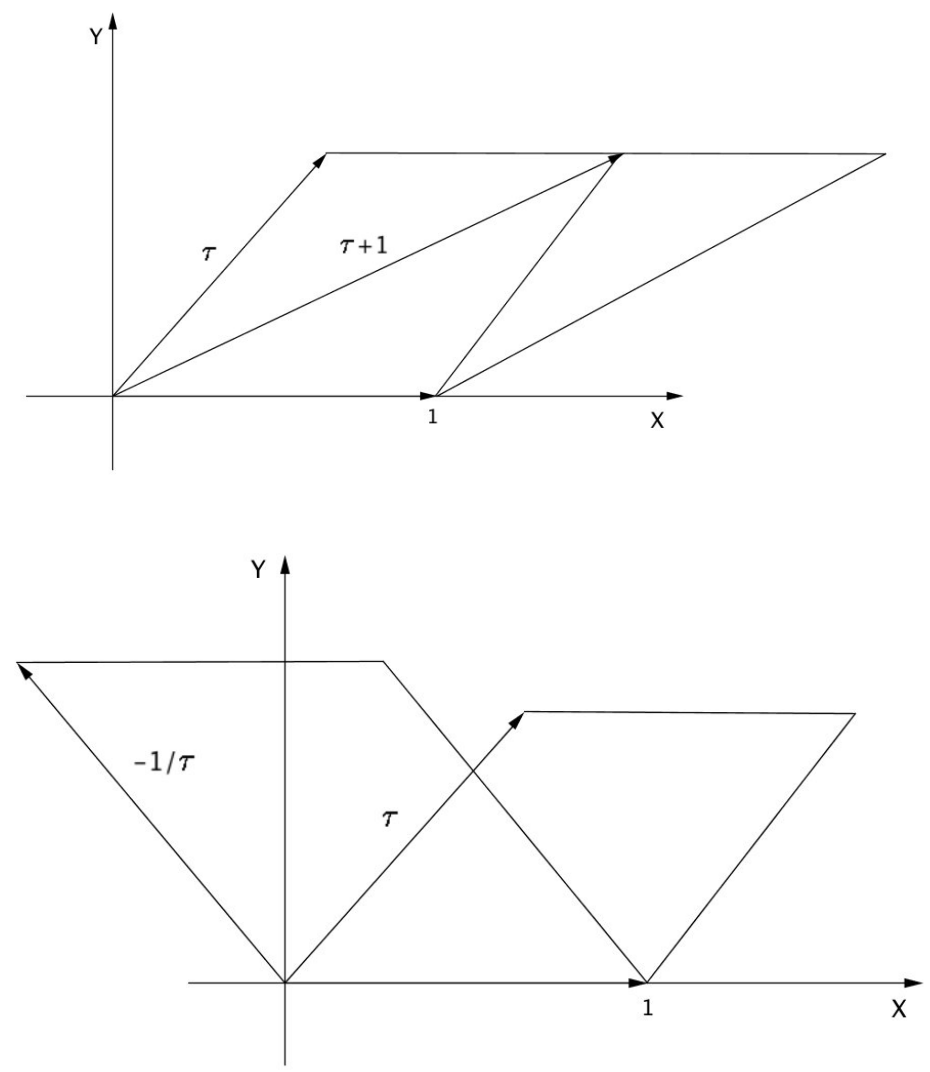

Figure 5. The modular transformations on the torus with modular parameter $\tau$.

in the semiclassical limit, as $\mathcal{I}(\mathcal{Q}(f)) \rightarrow I(f)$. Here and in the following, semiclassical limit means taking the inverse of the quantization map $\mathcal{Q}^{-1}(F)=f$ in the limit $\theta \rightarrow 0$, keeping only the leading contribution $[\cdot, \cdot] \rightarrow i\{\cdot, \cdot\}$ and dropping higher-order corrections in $\theta$. Sometimes this semi-classical limit is indicated by $F \rightarrow f$.

We are interested here in manifolds which can be realized as Poisson manifold $\mathcal{M}$ embedded in the Euclidean space $\mathbb{R}^{D}$, with Cartesian coordinates $x^{A}, A=1, \ldots, D$. The embedding is a map

$$
x^{A}: \mathcal{M} \hookrightarrow \mathbb{R}^{D},
$$

where $x^{A}$ are functions on $\mathcal{M}$. The Poisson tensor $\theta^{a b}$ is then defined via

$$
\left\{x^{A}, x^{B}\right\}=\theta^{a b} \partial_{a} x^{A} \partial_{b} x^{B} .
$$

A quantization of such a Poisson manifold provides in particular quantized embedding functions $x^{A}$ via

$$
X^{A}=\mathcal{Q}\left(x^{A}\right) \in \mathcal{A} \subset \operatorname{Mat}(\infty, \mathbb{C}) .
$$

Now consider the action for a scalar field $\Phi$ on such a quantized Poisson manifold in the matrix model, given by

$$
S=-\operatorname{Tr}\left(\left[X^{A}, \Phi\right]\left[X^{B}, \Phi\right] \delta_{A B}\right) .
$$

In the semiclassical limit $\Phi \sim \phi$, the action becomes

$$
S \sim \frac{1}{(2 \pi)^{n}} \int \mathrm{d}^{2 n} x \rho G^{a b} \partial_{a} \phi \partial_{b} \phi,
$$


where $\rho=\sqrt{\operatorname{det} \theta_{a b}^{-1}}$. Thus $G^{a b}=\theta^{a c} \theta^{b d} g_{c d}$ is identified as effective metric. In dimensions 4 or higher, this can be cast in the standard form for a scalar field coupled to a (conformally rescaled) metric [18]. In the present case of 2 dimensions this is not possible in general due to Weyl invariance, cf. [2]. However we are only considering tori with constant $\rho$ and $G^{a b}$ here, where this problem is irrelevant. Then $G^{a b}=e^{\sigma} g^{a b}$ as above is indeed the effective metric, up to possible conformal rescaling. Moreover, the matrix Laplace operator defined by

$$
\square \Phi:=\left[X^{A},\left[X^{B}, \Phi\right]\right] \delta_{A B}
$$

reduces in the semi-classical limit to

$$
\square \Phi \sim-g_{c d} \theta^{a c} \theta^{b d} \partial_{a} \partial_{b} \phi=-G^{a b} \partial_{a} \partial_{b} \phi=-\sqrt{|G|} \square_{G} \phi,
$$

where $\square_{G}$ is the standard Laplacian on manifold with metric $G^{a b}$. Thus the equation of motion for the scalar field reduces to

$$
\square_{G} \phi=0
$$

or equivalently $\square_{g} \phi=0$.

\subsection{The rectangular fuzzy torus in the matrix model}

The rectangular fuzzy torus can be defined in terms of two $N \times N$ unitary matrices, clock $C$ and shift $S$

$$
C=\left(\begin{array}{ccccc}
1 & & & & \\
& q & & & \\
& & q^{2} & & \\
& & & \ddots & \\
& & & & q^{N-1}
\end{array}\right), \quad S=\left(\begin{array}{ccccc}
0 & 1 & 0 & \cdots & 0 \\
0 & 0 & 1 & \cdots & 0 \\
& & \ddots & & \\
0 & & \cdots & 0 & 1 \\
1 & 0 & \cdots & & 0
\end{array}\right) .
$$

Here we introduce the deformation parameter $q=e^{i 2 \pi \theta}$, with phase $\theta=1 / N$ and positive integer $N \in \mathbb{N}$. The clock and shift matrices satisfy the relation

$$
C S=q S C
$$

and thus

$$
[C, S]=\left(1-q^{-1}\right) C S .
$$

These matrices are traceless and obey $C^{N}=S^{N}=1_{N}$. The fuzzy torus has a $\mathbb{Z}_{N} \times \mathbb{Z}_{N}$ symmetry, which acts on the algebra $\mathcal{A}_{N}$ as

$$
\begin{aligned}
& \mathbb{Z}_{N} \times \mathcal{A}_{N} \rightarrow \mathcal{A}_{N}, \\
& \left(\omega^{k}, \Phi\right) \mapsto C^{k} \Phi C^{-k}
\end{aligned}
$$

and similar for the other $\mathbb{Z}_{N}$ replacing $C$ by $S$. Here $\omega$ denotes the generator of $\mathbb{Z}_{N}$. Thus we have a decomposition of the algebra of function $\mathcal{A}_{N}$ over the torus into harmonics or irreducible representations of $\mathbb{Z}_{N} \times \mathbb{Z}_{N}$,

$$
\mathcal{A}_{N}=\bigoplus_{m, n=0}^{N-1} C^{n} S^{m} .
$$


An element in $\mathcal{A}_{N}$ can thus be written uniquely as

$$
\Phi(C, S)=\sum_{|n|,|m| \leq N / 2} c_{n m} q^{\frac{n m}{2}} C^{n} S^{m}
$$

This is hermitian $\Phi=\Phi^{\dagger}$ iff $c_{n m}=c_{-n,-m}^{*}$. The corresponding basis of functions on the classical torus is $e^{i n \sigma_{1}} e^{i m \sigma_{2}}$, for $n, m \in \mathbb{Z}$ and coordinates $\sigma_{1}, \sigma_{2} \in[0,2 \pi]$. Thus we obtain a quantization map from the functions on the torus to a matrix algebra

$$
\begin{aligned}
\mathcal{Q}: & \mathcal{C}\left(T^{2}\right) \rightarrow \mathcal{A}_{N}=M_{N}(\mathbb{C}), \\
e^{i n \sigma_{1}} e^{i m \sigma_{2}} & \mapsto \begin{cases}q^{\frac{n m}{2}} C^{n} S^{m}, & |n|,|m| \leq N / 2, \\
0, & \text { otherwise },\end{cases}
\end{aligned}
$$

which is one-to-one below the UV cutoff $n_{\max }, m_{\max }=N / 2$. This defines the fuzzy torus $T_{N}^{2}$. Now we consider the fuzzy torus embedded in $\mathbb{R}^{4}$, via the quantized embedding functions

$$
\begin{array}{ll}
X_{1}=\frac{R_{1}}{2}\left(C+C^{\dagger}\right), & X_{2}=-\frac{i R_{1}}{2}\left(C-C^{\dagger}\right), \\
X_{3}=\frac{R_{2}}{2}\left(S+S^{\dagger}\right), & X_{4}=-\frac{i R_{2}}{2}\left(S-S^{\dagger}\right) .
\end{array}
$$

The hermitian matrices $X_{1}, X_{2}, X_{3}$ and $X_{4}$ satisfy the algebraic relations

$$
X_{1}^{2}+X_{2}^{2}=R_{1}^{2}, \quad X_{3}^{2}+X_{4}^{2}=R_{2}^{2},
$$

which tells us that $R_{1}, R_{2}$ are the radii of the torus. This embedding defines derivations given by the adjoint action $\left[X_{i}, f\right]$ on $\mathcal{A}_{N}$.

Now consider the semi-classical limit. Then the clock and shift operators become plane waves, $C \rightarrow c=e^{i \sigma_{1}}$ and $S \rightarrow s=e^{i \sigma_{2}}$, where $\sigma_{a} \in[0,2 \pi]$. Observe that due to this periodicity, these $\sigma_{a}$ are standard coordinates on the torus as discussed before. We have then the embedding functions $x^{A}\left(\sigma_{1}, \sigma_{2}\right)$

$$
\begin{aligned}
& x^{1}=\frac{1}{2}\left(c+c^{\star}\right)=R_{1} \cos \left(\sigma_{1}\right), \\
& x^{2}=\frac{-i}{2}\left(c-c^{\star}\right)=R_{1} \sin \left(\sigma_{1}\right), \\
& x^{3}=\frac{1}{2}\left(s+s^{\star}\right)=R_{2} \cos \left(\sigma_{2}\right), \\
& x^{4}=\frac{-i}{2}\left(s-s^{\star}\right)=R_{2} \sin \left(\sigma_{2}\right),
\end{aligned}
$$

which again satisfy the algebraic relations

$$
\left(x^{1}\right)^{2}+\left(x^{2}\right)^{2}=R_{1}^{2}, \quad\left(x^{3}\right)^{2}+\left(x^{4}\right)^{2}=R_{2}^{2} .
$$

Using these embedding functions, we can compute the embedding (induced) metric

$$
g_{a b}=\frac{\partial x^{A}}{\partial \sigma^{a}} \frac{\partial x^{B}}{\partial \sigma^{b}} \delta_{A B}=\left(\begin{array}{cc}
R_{1}^{2} & 0 \\
0 & R_{2}^{2}
\end{array}\right)
$$

in standard coordinates. The Poisson structure is obtained from the semiclassical limit of the commutator

$$
[C, S]=\left(1-q^{-1}\right) C S \rightarrow \frac{i 2 \pi}{N} C S,
$$


where we expanded $q$ to first order of $1 / N$. On the other hand, classically we can write for the Poisson bracket

$$
\{c, s\}=\theta^{12} \partial_{1} c \partial_{2} s=-\theta^{12} c s .
$$

We can read off the Poisson tensor

$$
\theta^{c d}=\frac{2 \pi}{N}\left(\begin{array}{cc}
0 & -1 \\
1 & 0
\end{array}\right) .
$$

The corresponding symplectic structure is $\omega=\frac{N}{\pi} \mathrm{d} \sigma_{1} \wedge \sigma_{2}$. Given the embedding metric $g_{a b}$ and the Poisson tensor $\theta^{c d}$, we can compute the effective metric and the Laplacian. It is easy to see that in 2 dimensions, the effective metric $G^{a b}=\theta^{a c} \theta^{b d} g_{c d}$ is always proportional to the embedding metric $g_{a b}$ by a conformal rescaling

$$
G_{a b}=e^{-\sigma} g_{a b}
$$

For the Laplacian in 2 dimensions such conformal factors drop out, and indeed we have always identified conformally equivalent metrics on the torus. It is therefore sufficient here to work only with the embedding metric $g_{a b}$. With these tensors at hand, we can build the complex structure according to (3),

$$
J_{b}^{a}=\frac{\theta^{-1}}{\sqrt{g}} g_{b c} \theta^{c a}=\frac{1}{\sqrt{g}}\left(\begin{array}{cc}
0 & -R_{1}^{2} \\
R_{2}^{2} & 0
\end{array}\right)
$$

which satisfies $J^{2}=-1$, where $\theta^{-1}=\operatorname{det}\left(\theta_{a b}^{-1}\right)=\frac{N}{2 \pi}$. Since these are standard torus coordinates, we can read off the modular parameter which is purely imaginary,

$$
\tau=\frac{g_{12}+i \sqrt{g}}{g_{11}}=i \frac{R_{2}}{R_{1}} .
$$

Recalling that $\tau=\omega_{1} / \omega_{2}$, this corresponds to a rectangular torus with lattice vectors $\omega_{1}=i R_{2}$ and $\omega_{2}=R_{1}$.

\subsubsection{Laplacian of a scalar field}

Now consider a scalar field $\Phi \in \mathcal{A}_{N}$ on the basic fuzzy torus, with action (4)

$$
S=-\operatorname{Tr}\left[X^{A}, \Phi\right]\left[X^{B}, \Phi\right] \delta_{A B}
$$

and equation of motion $\square \Phi=0$. The matrix Laplacian operator (5) can be evaluated explicitly on the torus as

$$
\begin{aligned}
& 2 \square \Phi=\left[X^{A},\left[X^{B}, \Phi\right]\right] \delta_{A B}=R_{1}^{2}\left[C,\left[C^{\dagger}, \Phi\right]\right]+R_{2}^{2}\left[S,\left[S^{\dagger}, \Phi\right]\right] \\
& =R_{1}^{2}\left(2 \Phi-C \Phi C^{\dagger}-C^{\dagger} \Phi C\right)+R_{2}^{2}\left(2 \Phi-S \Phi S^{\dagger}-S^{\dagger} \Phi S\right), \\
& \square\left(C^{n} S^{m}\right)=c_{N}\left(R_{1}^{2}[n]_{q}^{2}+R_{2}^{2}[m]_{q}^{2}\right) C^{n} S^{m}, \\
& c_{N}=\left|q^{1 / 2}-q^{-1 / 2}\right|^{2} \rightarrow \frac{4 \pi^{2}}{N^{2}},
\end{aligned}
$$

where we have introduced the $q$-number

$$
[n]_{q}=\frac{q^{n / 2}-q^{-n / 2}}{q^{1 / 2}-q^{-1 / 2}}=\frac{\sin (n \pi / N)}{\sin (\pi / N)} \rightarrow n,
$$


so that

$$
[n]_{q}^{2}=\frac{q^{n}+q^{-n}-2}{q+q^{-1}-2}=\frac{\cos (2 n \pi / N)-1}{\cos (2 \pi / N)-1} \rightarrow n^{2} .
$$

In the semiclassical limit, the spectrum ${ }^{2}$ reduces to the spectrum of the commutative Laplacian

$$
\frac{4 \pi^{2}}{N^{2}}\left(R_{1}^{2} n^{2}+R_{2}^{2} m^{2}\right)
$$

\section{The fuzzy torus on a general lattice and fuzzy modular invariance}

To construct more general fuzzy tori, we define two unitary operators

$$
V_{x}\left(k_{x}, l_{x}\right)=C^{k_{x}} S^{l_{x}}, \quad V_{y}\left(k_{y}, l_{y}\right)=C^{k_{y}} S^{l_{y}},
$$

where $C$ and $S$ are the clock and shift matrix, and $k_{x}, l_{x}, k_{y}, l_{y} \in \mathbb{Z}$. The operators $V_{x}$ and $V_{y}$ generalize the clock and shift matrices, and satisfy $V_{x}^{N}=V_{y}^{N}=1$. Note that the $k_{x}, l_{x}, k_{y}, l_{y}$ should be considered more properly as elements of $\mathbb{Z}_{N}$, due to $C^{N}=S^{N}=1$. We combine these $k_{x}, l_{x}, k_{y}, l_{y}$ in two discrete complex vectors

$$
k=k_{x}+i k_{y} \in \mathbb{Z}_{N}+i \mathbb{Z}_{N} \equiv \mathbb{C}_{N}, \quad l=l_{x}+i l_{y} \in \mathbb{Z}_{N}+i \mathbb{Z}_{N} \equiv \mathbb{C}_{N},
$$

which define a lattice

$$
L_{N}(k, l)=\left\{n k+m l, n, m \in \mathbb{Z}_{N}\right\} .
$$

This is the fuzzy analogue of the lattice $L\left(\omega_{1}, \omega_{2}\right)$ which defines a commutative torus. The operators $V_{x}\left(k_{x}, l_{x}\right)$ and $V_{y}\left(k_{y}, l_{y}\right)$ satisfy the commutations relations

$$
V_{x} V_{y}=q^{k \wedge l} V_{y} V_{x}
$$

where

$$
k \wedge l=k_{x} l_{y}-k_{y} l_{x}
$$

is the area of the parallelogram spanned by $k$ and $l$. Note that the operators $V_{x}\left(k_{x}, l_{x}\right)$ and $V_{y}\left(k_{y}, l_{y}\right)$ commute if and only if $k \wedge l=0 \bmod N$, corresponding to collinear vectors spanning a degenerate torus, or tori whose area is a multiple of $N$.

Let us transform the lattice $L_{N}(k, l)$ with a $\operatorname{PSL}\left(2, \mathbb{Z}_{N}\right)=\operatorname{SL}\left(2, \mathbb{Z}_{N}\right) / \mathbb{Z}_{2}$ transformation to another lattice $L_{N}\left(k^{\prime}, l^{\prime}\right)$ :

$$
\left(\begin{array}{l}
k^{\prime} \\
l^{\prime}
\end{array}\right)=\left(\begin{array}{ll}
a & b \\
c & d
\end{array}\right)\left(\begin{array}{l}
k \\
l
\end{array}\right)
$$

Clearly the entries of the matrix should be elements of $\mathbb{Z}_{N}$, so that the transformed lattice vectors $k^{\prime}$ and $l^{\prime}$ are in $\mathbb{Z}_{N}$. On the $\operatorname{PSL}\left(2, \mathbb{Z}_{N}\right)$ transformed lattice $L_{N}\left(k^{\prime}, l^{\prime}\right)$ the commutation relations are

$$
V_{x}^{\prime} V_{y}^{\prime}=q^{k^{\prime} \wedge l^{\prime}} V_{y}^{\prime} V_{x}^{\prime}
$$

\footnotetext{
${ }^{2}$ It is interesting that the spectrum is the same as for a free boson in lattice theory, with lattice spacing $a=1 / N$.
} 
Since the area $k \wedge l$ is invariant under a $\operatorname{PSL}\left(2, \mathbb{Z}_{N}\right)$ transformation

$$
k^{\prime} \wedge l^{\prime}=(a d-b c) k \wedge l=k \wedge l
$$

it follows that this commutation relation is the same as for the original lattice

$$
V_{x}^{\prime} V_{y}^{\prime}=q^{k \wedge l} V_{y}^{\prime} V_{x}^{\prime}
$$

under the transformations (9). Thus we have established fuzzy modular invariance at the algebraic level, and we will consider noncommutative tori whose lattices are related by $\operatorname{PSL}\left(2, \mathbb{Z}_{N}\right)$ as equal. Later we will see that the spectrum of the Laplacian and the equation of motion for the noncommutative tori are also invariant under $\operatorname{PSL}\left(2, \mathbb{Z}_{N}\right)$. The moduli space of the lattice $L_{N}(k, l)$ or the fuzzy fundamental domain $\mathcal{F}_{N}$ is defined accordingly as

$$
\mathcal{F}_{N}=\mathbb{C}_{N} / \operatorname{PSL}\left(2, \mathbb{Z}_{N}\right)
$$

To obtain a metric structure, we define an embedding of these fuzzy tori into the $\mathbb{R}^{4}$ via the operators $V_{x}$ and $V_{y}$ as follows (cf. [12])

$$
\begin{aligned}
& X_{1}=\frac{R_{1}}{2}\left(V_{x}+V_{x}^{\dagger}\right)=\frac{R_{1}}{2}\left(C^{k_{x}} S^{l_{x}}+S^{-l_{x}} C^{-k_{x}}\right), \\
& X_{2}=-\frac{i R_{1}}{2}\left(V_{x}-V_{x}^{\dagger}\right)=-\frac{i R_{1}}{2}\left(C^{k_{x}} S^{l_{x}}-S^{-l_{x}} C^{-k_{x}}\right), \\
& X_{3}=\frac{R_{2}}{2}\left(V_{y}+V_{y}^{\dagger}\right)=\frac{R_{2}}{2}\left(C^{k_{y}} S^{l_{y}}+S^{-l_{y}} C^{-k_{y}}\right), \\
& X_{4}=-\frac{i R_{2}}{2}\left(V_{y}-V_{y}^{\dagger}\right)=-\frac{i R_{2}}{2}\left(C^{k_{y}} S^{l_{y}}-S^{-l_{y}} C^{-k_{y}}\right) .
\end{aligned}
$$

This embedding satisfies the algebraic relations $X_{1}^{2}+X_{2}^{2}=R_{1}^{2}$ and $X_{3}^{2}+X_{4}^{2}=R_{2}^{2}$ corresponding to two orthogonal $S^{1} \times S^{1}$. Nevertheless, the non-trivial ansatz for the $V_{x, y}$ will lead to a nontrivial effective geometry on the tori. As usual, this embedding defines derivations on the algebra $\mathcal{A}_{N}$ given by $\left[X_{i}, \cdot\right]$, and the integral is defined by the trace $\mathcal{I}(\Phi)=\frac{1}{N} \operatorname{Tr}(\Phi)$, where $\Phi$ denotes a scalar field on the torus

$$
\Phi=\sum_{\left(n_{1}, n_{2}\right) \in \mathbb{Z}_{N}^{2}} c_{n_{1} n_{2}} \Phi_{n_{1}, n_{2}} \in \mathcal{A}_{N}, \quad \Phi_{n_{1}, n_{2}}=q^{\frac{n_{1} n_{2}}{2}} C^{n_{1}} S^{n_{2}} .
$$

Here the momentum space is $\mathbb{Z}_{N}^{2} \cong[-N / 2+1, N / 2]^{2}$ if $N$ is even, to be specific. We are now ready to compute the spectrum of the Laplacian for a scalar field on the fuzzy torus,

$$
\begin{aligned}
& \square_{L_{N}} \Phi=\left[X^{A},\left[X^{B}, \Phi\right]\right] \delta_{A B}=R_{1}^{2}\left[V_{x},\left[V_{x}^{\dagger}, \Phi\right]\right]+R_{2}^{2}\left[V_{y},\left[V_{y}^{\dagger}, \Phi\right]\right] \\
& \quad=R_{1}^{2}\left(2 \Phi-V_{x} \Phi V_{x}^{\dagger}-V_{x}^{\dagger} \Phi V_{x}\right)+R_{2}^{2}\left(2 \Phi-V_{y} \Phi V_{y}^{\dagger}-V_{y}^{\dagger} \Phi V_{y}\right), \\
& \square_{L_{N}}\left(C^{n_{1}} S^{n_{2}}\right)=c_{N}\left(R_{1}^{2}\left[k_{x} n_{2}-l_{x} n_{1}\right]_{q}^{2}+R_{2}^{2}\left[k_{y} n_{2}-l_{y} n_{1}\right]_{q}^{2}\right) C^{n_{1}} S^{n_{2}}=: \lambda_{n_{1} n_{2}} C^{n_{1}} S^{n_{2}} .
\end{aligned}
$$

It is easy to see that this spectrum is invariant under the $\mathrm{SL}\left(2, \mathbb{Z}_{N}\right)$ modular transformations acting on the defining lattice $L_{N}(k, l)$ as in (9), and simultaneously on the momenta as follows

$$
\left(\begin{array}{l}
n_{1}^{\prime} \\
n_{2}^{\prime}
\end{array}\right)=\left(\begin{array}{ll}
a & b \\
c & d
\end{array}\right)\left(\begin{array}{l}
n_{1} \\
n_{2}
\end{array}\right) .
$$

Therefore fuzzy modular invariance is indeed a symmetry of fuzzy tori and their the scalar field spectrum. 


\subsection{Spectrum and Brillouin zone}

The above spectrum of $\square_{L_{N}}$ has a complicated periodicity structure, and typically some degeneracy in momentum space $\mathbb{C}_{N}$. In order to correctly identify the irreducible spectrum and the spectral geometry of the torus, we have to find the unit cell, or the first Brillouin zone $\mathcal{B}(\vec{s}, \vec{r})$. This unit cell is spanned by two vectors in momentum space

$$
\vec{r}=\left(r_{1}, r_{2}\right), \vec{s}=\left(s_{1}, s_{2}\right) \in \mathbb{Z}_{N}^{2},
$$

which characterize the basic periodicity of the spectrum. We can associate to them two elements $W_{r}=C^{r_{1}} S^{r_{2}}$ and $W_{s}=C^{s_{1}} S^{s_{2}}$ in $\mathcal{A}_{N}$. Then the shift in momentum space $\vec{n} \rightarrow \vec{n}+\vec{r}$ of the field $\Phi$ along $\vec{r}$ is realized by $\Phi W_{r}$, and the shift $\vec{n} \rightarrow \vec{n}+\vec{s}$ is realized by $\Phi W_{s}$. In order to compute these $\vec{s}$ and $\vec{r}$, we rewrite the spectrum in factorized form

$$
\begin{aligned}
\lambda_{n_{1} n_{2}}= & c_{N}\left(\left[k_{x} n_{2}-l_{x} n_{1}\right]_{q}^{2}+\left[k_{y} n_{2}-l_{y} n_{1}\right]_{q}^{2}\right) \\
= & 4\left(1-\cos \left[\frac{\pi}{N}\left(\left(k_{x}+k_{y}\right) n_{2}-\left(l_{x}+l_{y}\right) n_{1}\right)\right]\right. \\
& \left.\times \cos \left[\frac{\pi}{N}\left(\left(k_{x}-k_{y}\right) n_{2}-\left(l_{x}-l_{y}\right) n_{1}\right)\right]\right)
\end{aligned}
$$

using trigonometric identities, setting $R_{1}=R_{2}=1$ for simplicity. This allows to identify $\vec{r}$ as primitive periodicity of the first cos factor while leaving the second unchanged, and $\vec{s}$ as primitive periodicity of the second cos factor leaving the first unchanged. Explicitly,

$$
\begin{aligned}
& \cos \left[\frac{\pi}{N}\left(\left(k_{x}+k_{y}\right)\left(n_{2}+r_{2}\right)-\left(l_{x}+l_{y}\right)\left(n_{1}+r_{1}\right)\right)\right]=\cos \left[\frac{\pi}{N}\left(\left(k_{x}+k_{y}\right) n_{2}-\left(l_{x}+l_{y}\right) n_{1}\right)\right], \\
& \cos \left[\frac{\pi}{N}\left(\left(k_{x}-k_{y}\right)\left(n_{2}+s_{2}\right)-\left(l_{x}-l_{y}\right)\left(n_{1}+s_{1}\right)\right)\right]=\cos \left[\frac{\pi}{N}\left(\left(k_{x}-k_{y}\right) n_{2}-\left(l_{x}-l_{y}\right) n_{1}\right)\right] .
\end{aligned}
$$

This leads to the equations

$$
\left(k_{x}+k_{y}\right) r_{2}-\left(l_{x}+l_{y}\right) r_{1}=2 N, \quad\left(k_{x}-k_{y}\right) r_{2}-\left(l_{x}-l_{y}\right) r_{1}=0
$$

and

$$
\left(k_{x}+k_{y}\right) s_{2}-\left(l_{x}+l_{y}\right) s_{1}=0, \quad\left(k_{x}-k_{y}\right) s_{2}-\left(l_{x}-l_{y}\right) s_{1}=2 N .
$$

These four equations are equivalent to

$$
k_{x} r_{2}-l_{x} r_{1}=N, \quad k_{y} r_{2}-l_{y} r_{1}=N
$$

and

$$
k_{x} s_{2}-l_{x} s_{1}=N, \quad k_{y} s_{2}-l_{y} s_{1}=-N,
$$

which amount to $\left[V_{x, y}, W_{r, s}\right]=0$. In complex notation, these 4 equations can be written as

$$
k r_{2}-l r_{1}=N(1+i), \quad k s_{2}-l s_{1}=N(1-i)
$$

or in matrix form

$$
\left(\begin{array}{l}
1+i \\
1-i
\end{array}\right)=\frac{1}{N}\left(\begin{array}{ll}
r_{2} & -r_{1} \\
s_{2} & -s_{1}
\end{array}\right)\left(\begin{array}{l}
k \\
l
\end{array}\right)
$$

In particular, this implies

$$
2 N^{2}=|\vec{r} \wedge \vec{s}||k \wedge l|,
$$


reflecting the decomposition of the momentum space $\mathbb{Z}_{N}^{2}$ into Brillouin zones. Alternatively, these equations can be written as

$$
\left(\begin{array}{l}
1+i \\
1-i
\end{array}\right)=\frac{1}{N}\left(\begin{array}{ll}
k_{x} & -l_{x} \\
k_{y} & -l_{y}
\end{array}\right)\left(\begin{array}{l}
b \\
a
\end{array}\right)
$$

introducing the following complex combinations

$$
a=r_{1}+i s_{1}, b=r_{2}+i s_{2} \in \mathbb{C}_{N} .
$$

Inverting (13) gives

$$
\left(\begin{array}{l}
k \\
l
\end{array}\right)=\frac{N}{r_{1} s_{2}-r_{2} s_{1}}\left(\begin{array}{ll}
-s_{1} & r_{1} \\
-s_{2} & r_{2}
\end{array}\right)\left(\begin{array}{c}
1+i \\
1-i
\end{array}\right) \text {. }
$$

However, all quantities in these equations must be integers in $\left[-\frac{N}{2}, \frac{N}{2}\right]$, to be specific. Therefore non-trivial Brillouin zones $\mathcal{B}(\vec{s}, \vec{r})$ are typically possible only if their area $|\vec{r} \wedge \vec{s}|=r_{1} s_{2}-r_{2} s_{1}$ divides $^{3} N$. Similarly, inverting (15) gives

$$
\left(\begin{array}{l}
b \\
a
\end{array}\right)=\frac{N}{k_{y} l_{x}-k_{x} l_{y}}\left(\begin{array}{cc}
-l_{y} & l_{x} \\
-k_{y} & k_{x}
\end{array}\right)\left(\begin{array}{c}
1+i \\
1-i
\end{array}\right)
$$

and again $|k \wedge l|=k_{y} l_{x}-k_{x} l_{y}$ must typically divide $N$.

The above analysis leads to a very important point. The equations (17) which determine the first Brillouin zone are Diophantic equations, so that their naive solutions in $\mathbb{R}^{2}$ may not be admissible in $\mathbb{C}_{N}$. This follows also from (14), which is very restrictive e.g. if $N$ is a prime number. If (17) gives non-integer $(r, s)$ for given $(k, l)$, then these naive Brillouin zones and their apparent spectral geometry are not physical; in that case, the full spectrum obtained by properly organizing all physical modes in momentum space $\left(n_{1}, n_{2}\right)$ may look very different. To see this, consider $N$ prime and $k, l$ relatively prime. Then there are unitary operators $\tilde{C}=V_{x}^{n}$, $\tilde{S}=V_{y}^{m}$ which generate $\mathcal{A}_{N}$ with $V_{x} \tilde{C}=q \tilde{C} V_{x}$ and $V_{y} \tilde{S}=q^{-1} \tilde{C} V_{y}$, leading to the spectral geometry (7) of a rectangular torus; this is in contrast to (16) which falsely suggests a nontrivial lattice and Brillouin zone. On the other hand, if $N$ is divisible by $\left(k_{y} l_{x}-k_{x} l_{y}\right)$, then the above equations (17) can be solved for $a, b \in \mathbb{C}_{N}$, for any given non-trivial lattice $L_{N}(k, l)$. In that case, we obtain indeed a fuzzy version of the desired non-trivial torus as discussed below, with periodic spectrum decomposing into several isomorphic Brillouin zones $\mathcal{B}(\vec{s}, \vec{r})$.

To illustrate this, we choose a lattice $L_{N}(k, l)$ with vectors $l=2+i$ and $k=2+4 i$, with area $k \wedge l=6$. The smallest matrix size to accommodate this is $N=6$, and in this case the corresponding Brillouin zone $B(\vec{r}, \vec{s})$ is spanned by $\vec{r}=-2+i, \vec{s}=-6-3 i$ with $\vec{r} \wedge \vec{s}=12$, see Fig. 6. Thus momentum space decomposes into 3 copies of the Brillouin zone.

\subsection{Effective geometry}

Now we want to understand the effective geometry of the torus $L_{N}(k, l)$ in the semi-classical limit. We will discuss both the spectral geometry as well as the effective geometry in the sense of Section 3, which should of course agree. In the semi-classical limit, we would like that the integers $k_{x}, l_{x}, k_{y}, l_{y}$ approach in some sense the real numbers $\omega_{1 x}, \omega_{2 x}, \omega_{1 y}, \omega_{2 y}$ corresponding to some generic classical torus. More precisely, the lattice $L_{N}(k, l)$ should approach some given lattice $L\left(\omega_{1}, \omega_{2}\right)$. This can be achieved via a sequence of rational numbers approximating these real numbers. Explicitly, we require

$$
\frac{k_{N}}{\rho_{N}} \rightarrow \omega_{1}, \quad \frac{l_{N}}{\rho_{N}} \rightarrow \omega_{2},
$$

\footnotetext{
${ }^{3}$ This condition may be avoided e.g. if the $r_{i}, s_{i}$ are not relatively prime.
} 


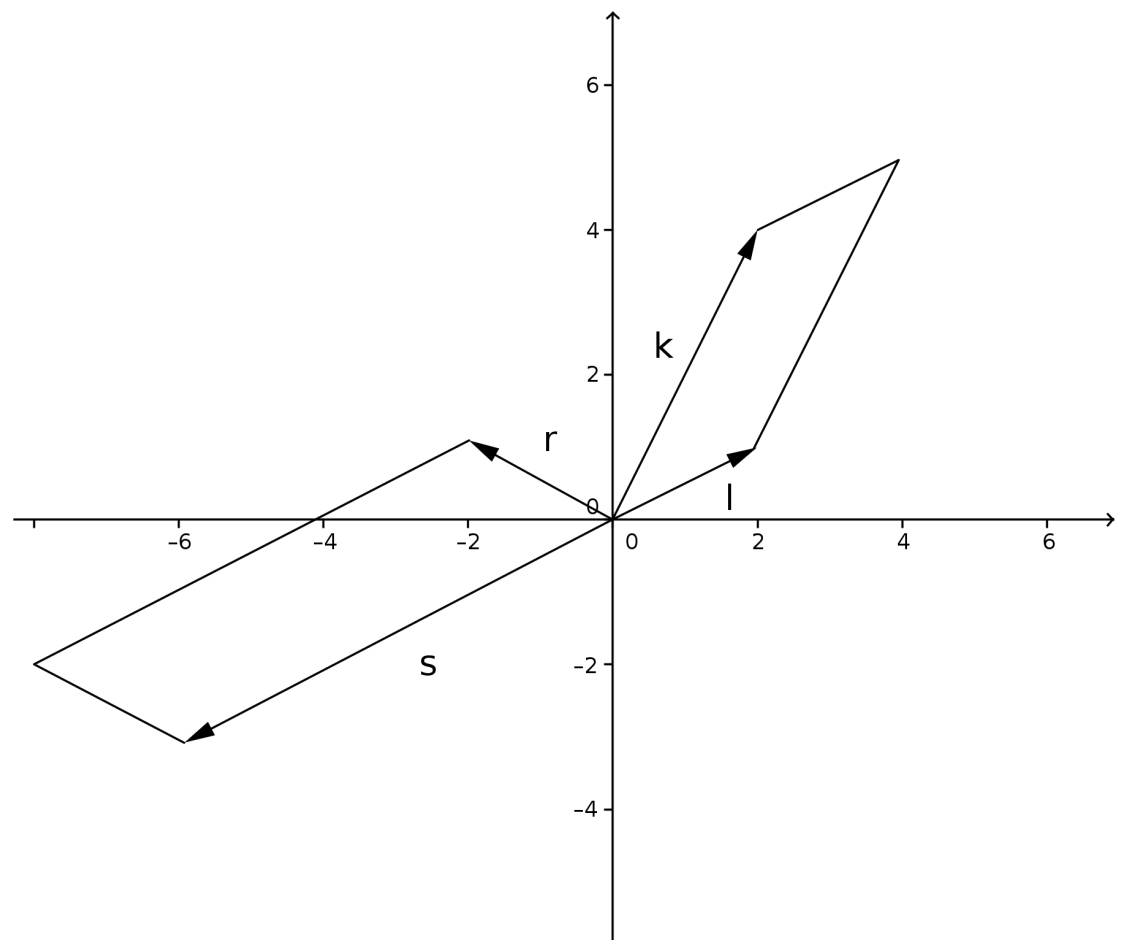

Figure 6. The upper parallelogram spanned by the vectors $k$ and $l$ is the geometric torus. The lower parallelogram is the unit cell $\mathcal{B}(\vec{r}, \vec{s})$.

where $\rho_{N}$ is some increasing function of $N$. Now consider the spectrum

$$
\begin{aligned}
\lambda_{n_{1} n_{2}} & =4 \sin ^{2}\left(\frac{\pi}{N}\left(k_{x} n_{2}-l_{x} n_{1}\right)\right)+4 \sin ^{2}\left(\frac{\pi}{N}\left(k_{y} n_{2}-l_{y} n_{1}\right)\right) \\
& \rightarrow\left(\frac{2 \pi \rho_{N}}{N}\right)^{2}\left|\omega_{1} n_{2}-\omega_{2} n_{1}\right|^{2}
\end{aligned}
$$

setting $R_{1}=R_{2}=1$. This approximation is valid as long as the argument of the $\sin ()$ terms are smaller than one, i.e. in the interior of the first Brillouin zone. As we will verify below, this spectrum indeed reproduces the spectrum of the classical Laplace operator on the torus $L\left(\omega_{1}, \omega_{2}\right)$ in the semi-classical limit $N \rightarrow \infty$, as long as $\left|\omega_{1} n_{2}-\omega_{2} n_{1}\right|<\frac{N}{\rho_{N}}$.

Now consider the effective geometry in the semi-classical limit, as discussed in Section 3. Since $C \sim e^{i \sigma_{1}}$ and $S \sim e^{i \sigma_{2}}$, the defining matrices $V_{x}$ and $V_{y}(8)$ of the fuzzy torus $L_{N}(k, l)$ become

$$
V_{x} \sim v_{x}=e^{i\left(\tilde{\sigma}_{1} \omega_{1 x}+\tilde{\sigma}_{2} \omega_{2 x}\right)}, \quad V_{y} \sim v_{y}=e^{i\left(\tilde{\sigma}_{1} \omega_{1 y}+\tilde{\sigma}_{2} \omega_{2 y}\right)}
$$

Here $\tilde{\sigma}_{1,2}=\rho_{N} \sigma_{i}$ are defined on $\left[0,2 \pi \rho_{N}\right]$. The Poisson brackets can be obtained from

$$
\left[V_{x}, V_{y}\right] \sim \frac{2 \pi}{N} k \wedge l v_{x} v_{y} \rightarrow \frac{2 \pi \rho_{N}^{2}}{N}\left(\omega_{1 x} \omega_{2 x}-\omega_{1 y} \omega_{2 y}\right) v_{x} v_{y}
$$

The semi-classical approximation makes sense as long as $k \wedge l<N$, which holds for at least one equivalent torus $L_{N}\left(k^{\prime}, l^{\prime}\right)$ if $\mathbb{C}_{N}$ decomposes into at least $N$ fundamental domains $\mathcal{F}_{N}(10)$. We can then identify this with the Poisson bracket

$$
\left\{v_{x}, v_{y}\right\}=\tilde{\theta}^{12}\left(\omega_{1 x} \omega_{2 x}-\omega_{1 y} \omega_{2 y}\right) v_{x} v_{y},
$$


and read off the Poisson tensor for the $\tilde{\sigma}_{i}$ coordinates

$$
\left\{\tilde{\sigma}^{a}, \tilde{\sigma}^{b}\right\}=\tilde{\theta}^{a b}=\frac{2 \pi \rho_{N}^{2}}{N}\left(\begin{array}{cc}
0 & -1 \\
1 & 0
\end{array}\right) .
$$

The embedding functions in $\mathbb{R}^{4}$ become

$$
\begin{aligned}
& x_{1}=\frac{R_{1}}{2}\left(v_{x}+v_{x}^{\star}\right)=R_{1} \cos \left(\tilde{\sigma}_{1} \omega_{1 x}+\tilde{\sigma}_{2} \omega_{2 x}\right), \\
& x_{2}=\frac{-i R_{1}}{2}\left(v_{x}-v_{x}^{\star}\right)=R_{1} \sin \left(\tilde{\sigma}_{1} \omega_{1 x}+\tilde{\sigma}_{2} \omega_{2 x}\right), \\
& x_{3}=\frac{R_{2}}{2}\left(v_{y}+v_{y}^{\star}\right)=R_{2} \cos \left(\tilde{\sigma}_{1} \omega_{1 y}+\tilde{\sigma}_{2} \omega_{2 y}\right), \\
& x_{4}=\frac{-i R_{2}}{2}\left(v_{y}-v_{y}^{\star}\right)=R_{2} \sin \left(\tilde{\sigma}_{1} \omega_{1 y}+\tilde{\sigma}_{2} \omega_{2 y}\right)
\end{aligned}
$$

and satisfy again the algebraic relations

$$
x_{1}^{2}+x_{2}^{2}=R_{1}^{2}, \quad x_{3}^{2}+x_{4}^{2}=R_{2}^{2} .
$$

The embedding metric is computed via (6),

$$
\begin{aligned}
\mathrm{d} s^{2}= & \left(\left(\omega_{1 x} R_{1}\right)^{2}+\left(\omega_{1 y} R_{2}\right)^{2}\right)\left(\mathrm{d} \tilde{\sigma}^{1}\right)^{2}+2\left(\omega_{1 x} \omega_{2 x} R_{1}^{2}+\omega_{1 y} \omega_{2 y} R_{2}^{2}\right) \mathrm{d} \tilde{\sigma}^{1} \mathrm{~d} \tilde{\sigma}^{2} \\
& +\left(\left(\omega_{2 x} R_{1}\right)^{2}+\left(\omega_{2 y} R_{2}\right)^{2}\right)\left(\mathrm{d} \tilde{\sigma}^{2}\right)^{2} .
\end{aligned}
$$

This reproduces indeed the metric of the general torus $L\left(\omega_{1}, \omega_{2}\right)$ (1) for $R_{1}=R_{2}=1$, which is recovered here from a series of fuzzy tori $L_{N}\left(k_{N}, l_{N}\right)$.

As a consistency check, we compute the spectrum of the commutative Laplacian and compare it with the semiclassical limit (4.2). Since $G_{a b} \sim g_{a b}$ in 2 dimensions as discussed before, the Laplacian is proportional to

$$
\square=g^{a b} \partial_{a} \partial_{b}=\left(\omega_{1 x}^{2}+\omega_{1 y}^{2}\right) \partial_{\sigma_{1}}^{2}+2\left(\omega_{1 x} \omega_{2 x}+\omega_{1 y} \omega_{2 y}\right) \partial_{\sigma_{1}} \partial_{\sigma_{2}}+\left(\omega_{2 x}^{2}+\omega_{2 y}^{2}\right) \partial_{\sigma_{1}}^{2}
$$

setting $R_{1}=R_{2}=1$ and dropping the tilde on $\sigma_{i}$. Evaluating this on $e^{i n \sigma_{1}} e^{i m \sigma_{2}}$ we obtain

$$
\begin{aligned}
\square e^{i n \sigma_{1}} e^{i m \sigma_{2}} & =\left[\left(\omega_{1 x}^{2}+\omega_{1 y}^{2}\right) n^{2}+2\left(\omega_{1 x} \omega_{2 x}+\omega_{1 y} \omega_{2 y}\right) n^{2} m^{2}+\left(\omega_{2 x}^{2}+\omega_{2 y}^{2}\right) m^{2}\right] e^{i n \sigma_{1}} e^{i m \sigma_{2}} \\
& =\left|\omega_{1} m-\omega_{2} n\right|^{2} e^{i n \sigma_{1}} e^{i m \sigma_{2}} .
\end{aligned}
$$

This agrees (up to an irrelevant factor) with the semiclassical spectrum (4.2) of the matrix Laplacian.

Given the metric and the Poisson structure, we can compute the complex structure

$$
J_{b}^{a}=\frac{\tilde{\theta}^{-1}}{\sqrt{g}} g_{b c} \tilde{\theta}^{c a}=\frac{1}{\sqrt{g}}\left(\begin{array}{ll}
g_{12} & -g_{11} \\
g_{22} & -g_{12}
\end{array}\right)
$$

which satisfies $J^{2}=-1$. Here $\tilde{\theta}^{-1}=\operatorname{det}\left(\tilde{\theta}_{a b}^{-1}\right)$. The effective modular parameter in the commutative case is given by $\tau=\omega_{1} / \omega_{2} \in \mathcal{F}$. In the fuzzy case, we can choose a sequence of moduli parameter depending on $N$

$$
\tau_{N}=\frac{k_{N}}{l_{N}} \in \mathbb{C}_{N}
$$

which for $N \rightarrow \infty$ approximates the complex number $\tau$ to arbitrary precision. 
Finally let us discuss the quantization map. There is a natural map

$$
\begin{aligned}
\mathcal{Q}: & \mathcal{C}\left(T^{2}\right) \rightarrow \mathcal{A}_{N}=M_{N}(\mathbb{C}), \\
e^{i n_{1} \sigma_{1}} e^{i n_{2} \sigma_{2}} & \mapsto \begin{cases}q^{\frac{n_{1} n_{2}}{2}} C^{n_{1}} S^{n_{2}}, & \left|n_{i}\right| \leq \frac{N}{2}, \\
0, & \text { otherwise, }\end{cases}
\end{aligned}
$$

where $n_{1}, n_{2} \in \mathbb{Z}$, and $\sigma_{1}, \sigma_{2} \in[0,2 \pi]$ are coordinates on $T^{2}$, which respects the harmonic decomposition with respect to the classical and matrix Laplacians. In particular,

$$
\mathcal{Q}\left(e^{i\left(\omega_{1 x} \tilde{\sigma}_{1}+\omega_{2 x} \tilde{\sigma}_{2}\right)}\right)=C^{k_{x}} S^{l_{x}}=V_{x}, \quad \mathcal{Q}\left(e^{i\left(\omega_{1 y} \tilde{\sigma}_{1}+\omega_{2 y} \tilde{\sigma}_{2}\right)}\right)=C^{k_{y}} S^{l_{y}}=V_{y}
$$

(up to phase factors) with

$$
\omega_{1} \rho_{N} \approx k, \quad \omega_{2} \rho_{N} \approx l .
$$

Now assume that (17) is solved by integers $r_{i}, s_{i}$, defining the Brillouin zone $\mathcal{B}(\vec{r}, \vec{s})$. Then the spectrum of $\square$ is $n$-fold degenerate, and (19) describes the quantization of an $n$-fold covering of the basic torus. Indeed the elements $W_{r}, W_{s}$ generate a discrete group $\mathcal{G}_{W} \subset U(N)$ acting on $\mathcal{A}_{N}$ from the right, which leaves $\square$ invariant and permutes the different tori resp. Brillouin zones. Accordingly, the space of functions on a single fuzzy torus $L_{N}(k, l)$ is given by the quotient $\tilde{A}_{N}=M_{N}(\mathbb{C}) / \mathcal{G}_{W}$, which is a vector space rather than an algebra. Nevertheless, it is natural to consider the map

$$
\begin{aligned}
& \tilde{\mathcal{Q}}: \quad \mathcal{C}\left(T^{2}\right) \rightarrow \tilde{\mathcal{A}}_{N}=M_{N}(\mathbb{C}) / \mathcal{G}_{W}, \\
& e^{i n_{1} \sigma_{1}} e^{i n_{2} \sigma_{2}} \mapsto \begin{cases}q^{\frac{n_{1} n_{2}}{2}} C^{n_{1}} S^{n_{2}}, & \left(n_{1}, n_{2}\right) \in \mathcal{B}(\vec{r}, \vec{s}), \\
0, & \text { otherwise }\end{cases}
\end{aligned}
$$

as quantization of the torus $L\left(\omega_{1}, \omega_{2}\right)$ under consideration.

\subsection{Partition function}

The partition function for a scalar field on the fuzzy torus as discussed in Section 3.1.1 is defined via the functional approach as

$$
Z_{N}(k, l)=\int D \Phi e^{-\Phi \square \Phi}=\int \mathrm{d} \phi_{n m} \mathrm{~d} \phi_{n^{\prime} m^{\prime}} e^{-c_{N} \sum_{n m ; n^{\prime} m^{\prime}} \phi_{n m} \Omega_{n n^{\prime} ; m m^{\prime}} \phi_{n^{\prime} m^{\prime}}}
$$

with $Q_{n m}=\left[k_{x} m-l_{x} n\right]^{2}+\left[k_{y} m-l_{y} n\right]^{2}$ and $\Omega_{n n^{\prime} ; m m^{\prime}}=\delta_{n n^{\prime}} \delta_{m m^{\prime}}\left(Q_{n m}+\epsilon\right)$. Here $D \Phi$ denotes the standard measure on the space of hermitian $N \times N$ matrices, and $\epsilon$ is a small number introduced to regularize the divergence due to the zero modes. The Gaussian integral gives

$$
Z_{N}(k, l)=\frac{1}{\left.\sqrt{\operatorname{det}\left(Q_{n m}+\epsilon\right.}\right)}=\epsilon^{-1 / 2} \prod_{n, m \neq 0}^{N-1}\left(\left[k_{x} m-l_{x} n\right]^{2}+\left[k_{y} m-l_{y} n\right]^{2}+\epsilon\right)^{-1 / 2} .
$$

We renormalize the partition function by multiplying with $\epsilon^{1 / 2}$, and after taking the limit $\epsilon \rightarrow 0$ we find

$$
Z_{N}(k, l)=\prod_{n, m \neq 0}^{N-1}\left(\left[k_{x} m-l_{x} n\right]^{2}+\left[k_{y} m-l_{y} n\right]^{2}\right)^{-1 / 2}
$$

This is completely well-defined, and invariant under the fuzzy modular group $\operatorname{SL}\left(2, \mathbb{Z}_{N}\right)$

$$
Z_{N}\left(k^{\prime}, l^{\prime}\right)=Z(k, l)
$$


using the above results. For example, the partition function for the rectangular fuzzy torus corresponds to the lattice $k_{y}=l_{x}=1$ and $k_{x}=l_{y}=0$,

$$
Z_{N}(1, i)=\prod_{n, m \neq 0}^{N-1}\left([n]^{2}+[m]^{2}\right)^{-1 / 2}
$$

In the limit $N \rightarrow \infty$, the partition function (20) looks very similar to the partition function of the commutative torus $L\left(\omega_{1}, \omega_{2}\right) \cong L(\tau, 1)$, which up to a factor takes the form

$$
\begin{aligned}
Z\left(\omega_{1}, \omega_{2}\right) & =\prod_{n, m \neq 0}^{\infty}\left(\left(\omega_{1 x} m-\omega_{2 x} n\right)^{2}+\left(\omega_{1 y} m-\omega_{2 y} n\right)^{2}\right)^{-1 / 2} \\
& =\left(\prod_{n, m \neq 0}^{\infty}(\tau m+n)(\bar{\tau} m+n)\right)^{-1 / 2}
\end{aligned}
$$

However $Z_{N}$ provides a regularization which is not equivalent to a simple cutoff or zeta function regularization (see for example [17]), because the spectrum of the fuzzy torus significantly differs from the commutative one near the boundary of the Brillouin zone, thus regularizing the theory. Moreover, there may be some multiplicity due to the periodic structure of Brillouin zones.

Similarly, the free energy for a scalar field on the fuzzy torus is obtained from the partition function via

$$
\begin{aligned}
F_{N}=\ln Z_{N}= & -\frac{1}{2} \sum_{n_{1}, n_{2} \neq 0}^{N-1} \ln \left[\sin ^{2}\left(\frac{\pi}{N}\left(k_{x} n_{2}-l_{x} n_{1}\right)\right)+\sin ^{2}\left(\frac{\pi}{N}\left(k_{y} n_{2}-l_{y} n_{1}\right)\right)\right] \\
= & -\frac{1}{2} \sum_{n_{1}, n_{2} \neq 0}^{N-1} \ln \left[\left(1-\cos \left(\frac{\pi}{N}\left(\left(k_{x}+k_{y}\right) n_{2}-\left(l_{x}+l_{y}\right) n_{1}\right)\right)\right.\right. \\
& \left.\times \cos \left(\frac{\pi}{N}\left(\left(k_{x}-k_{y}\right) n_{2}-\left(l_{x}-l_{y}\right) n_{1}\right)\right)\right]
\end{aligned}
$$

using the identity (12). In the semi-classical approximation

$$
\frac{k}{\rho_{N}} \rightarrow \omega_{1}, \quad \frac{l}{\rho_{N}} \rightarrow \omega_{2}
$$

we can replace the sum by an integral

$$
\begin{aligned}
F\left(\omega_{1}, \omega_{2}\right)= & -\frac{\mathcal{N}}{2} \int_{\mathcal{B}\left(\omega_{1}, \omega_{2}\right)} \mathrm{d} \sigma_{1} \mathrm{~d} \sigma_{2} \ln \left[\left(1-\cos \left(\pi\left(\left(\omega_{1 x}+\omega_{1 y}\right) \sigma_{1}-\left(\omega_{2 x}+\omega_{2 y}\right) \sigma_{2}\right)\right)\right)\right. \\
& \left.\times \cos \left(\pi\left(\left(\omega_{1 x}-\omega_{1 y}\right) \sigma_{1}+\left(\omega_{2 x}-\omega_{2 y}\right) \sigma_{2}\right)\right)\right]
\end{aligned}
$$

over the appropriate Brillouin zone, where $\mathcal{N}$ denotes its multiplicity. This integral is invariant under $\operatorname{SL}(2, \mathbb{R})$ transformation of the lattice vectors $\omega_{1}$ and $\omega_{2}$. However we have not been able to evaluate it in closed form.

We conclude with some remarks on possible applications of the above results. In the context of string theory, a natural problem is to integrate over the moduli space of all tori. This arises e.g. in the computation of the one-loop partition function of the bosonic string. The fuzzy torus regularization should provide a useful new tool to address this type of problem, taking advantage of its bounded spectrum and discretized moduli space. The integration over the moduli space of all tori corresponds here to the sum of the partition function (20) over all fuzzy tori defined 
by $k$ and $l$. This is certainly finite for any given $N$, since the moduli space $\mathbb{Z}_{N}^{2}$ is finite. To define the sum over all tori, there are two natural prescriptions. First, one can consider

$$
\mathcal{Z}=\sum_{\mathbb{Z}_{N}^{2}} Z_{N}(k, l)
$$

This of course entails an over-counting of lattices $L_{N}(l, k)$ related by $\operatorname{SL}\left(2, \mathbb{Z}_{N}\right)$, but it is still finite. On the other hand, one could compute

$$
\mathcal{Z}^{\prime}=\sum_{\mathbb{Z}_{N}^{2} / \mathrm{SL}\left(2, \mathbb{Z}_{N}\right)} Z_{N}(k, l)
$$

which is analogous to the one-loop partition function for a closed bosonic string [17]. If all $\mathrm{SL}\left(2, \mathbb{Z}_{N}\right)$ orbits on $\mathbb{Z}_{N}^{2}$ have the same cardinality, then the two definitions for $\mathcal{Z}$ and $\mathcal{Z}^{\prime}$ are related by a factor and hence equivalent. However this may not be true in general, and the two definitions may not be equivalent in the large $N$ limit. We leave a more detailed study of these issues to future work.

Finally, the form of the spectrum of the Laplacian on $L_{N}(l, k)$ suggests to formulate a finite analog of the modular form $E\left(1, \omega_{1}, \omega_{2}\right)$

$$
E\left(1, \omega_{1}, \omega_{2}\right)=\sum_{n, m \neq 0}^{\infty} \frac{1}{\left(\omega_{1} n+\omega_{2} m\right)^{2}},
$$

which could be replaced here by the fuzzy analog

$$
E_{q}(1, l, k)=\sum_{n, m \in \mathcal{B}(\vec{r}, \vec{s}) \backslash\{0\}} \frac{1}{\left[k_{x} m-l_{x} n\right]_{q}^{2}+\left[k_{y} m-l_{y} n\right]_{q}^{2}} .
$$

This is invariant under $\operatorname{PSL}\left(2, \mathbb{Z}_{N}\right)$, and reduces to $\left(\frac{2 \pi \rho_{N}}{N}\right)^{2} E\left(1, \omega_{1}, \omega_{2}\right)$ in the limit $N \rightarrow \infty$. It would be interesting to construct fuzzy $E_{q}(p, l, k)$ which reduce to Eisenstein series $E\left(p, \omega_{1}, \omega_{2}\right)$ in the limit $N \rightarrow \infty$.

\subsection{The general fuzzy tori as solution of the massive matrix model}

It is easy to see that the general torus corresopnding to the lattice $L_{N}$ as above is a solution of the massive matrix model with equations of motion

$$
\square_{L_{N}} X^{A}=\lambda X^{A}
$$

as observed in [12]. Using the matrices (11) we find

$$
\square_{L_{N}} X^{A}=4 R_{i}^{2} \sin ^{2}\left(\frac{2 \pi\left(k_{x} l_{y}-k_{y} l_{x}\right)}{N}\right) X^{A}=c_{N} R_{i}^{2}\left[\left(k_{x} l_{y}-k_{y} l_{x}\right)\right]_{q}^{2} X^{A}
$$

with $i=2$ for $A=1,2$ and $i=1$ for $A=3,4$. Thus the embedding function $X^{a}$ are solutions of (21) for $R_{1}=R_{2}=R$ and

$$
c_{N} R^{2}\left[\left(k_{x} l_{y}-k_{y} l_{x}\right)\right]_{q}^{2}=\lambda,
$$

where $c_{N}$ is defined in (7). The spectrum is invariant under $\operatorname{SL}\left(2, \mathbb{Z}_{N}\right)$ transformation, as shown before. In the semiclassical limit, the equations of motion reduce to

$$
\square_{L} x^{A}=\left(\frac{2 R \rho_{N}}{N}\right)^{2}\left(\bar{\omega}_{1} \omega_{2}-\omega_{1} \bar{\omega}_{2}\right)^{2} x^{A}
$$

or $\square_{G} x^{A} \sim-\tau_{2}^{2} x^{A}$ if the lattice vectors are chosen to be $\omega_{1}=\tau$ and $\omega_{2}=1$. 


\subsection{Dirac operator on the fuzzy torus}

In this final section we briefly discuss the Dirac equation on the rectangular fuzzy torus generated by $C$ and $S$. As usual in matrix models $[5,13,18]$, the matrix Dirac operator $\not D$ is based on the Clifford algebra of the embedding space, which is 4-dimensional here. Although this $\not D$ is in general not equivalent to the standard Dirac operator on a Riemannian manifold, a relation can typically be established at least in the semi-classical limit $N \rightarrow \infty$ by applying some projection operator, as elaborated in several examples [1,9]. Here we only study the spectrum of $\not D$ at finite $N$.

First, we introduce the following representation of the two-dimensional Euclidean Gamma matrices

$$
\gamma^{0}=\left(\begin{array}{cc}
0 & i \\
-i & 0
\end{array}\right), \quad \gamma^{1}=\left(\begin{array}{cc}
0 & 1 \\
1 & 0
\end{array}\right)
$$

which satisfy the Clifford algebra $\left\{\gamma^{i}, \gamma^{j}\right\}=2 \delta^{i j}$. Then a 4-dimensional Clifford algebra can then be constructed as follows

$$
\begin{array}{ll}
\Gamma^{0}=\gamma^{0} \otimes\left(\begin{array}{cc}
-1 & 0 \\
0 & 1
\end{array}\right), \quad \Gamma^{1}=\gamma^{1} \otimes\left(\begin{array}{cc}
-1 & 0 \\
0 & 1
\end{array}\right), \\
\Gamma^{2}=I \otimes\left(\begin{array}{ll}
0 & 1 \\
1 & 0
\end{array}\right), \quad \Gamma^{3}=I \otimes\left(\begin{array}{cc}
0 & -i \\
i & 0
\end{array}\right) .
\end{array}
$$

Now we define

$$
\begin{array}{lll}
\Gamma_{+}^{1}=\frac{1}{2}\left(\Gamma^{0}+i \Gamma^{1}\right), & \Gamma_{-}^{1}=\frac{1}{2}\left(\Gamma^{0}-i \Gamma^{1}\right), \\
\Gamma_{+}^{2}=\frac{1}{2}\left(\Gamma^{2}+i \Gamma^{3}\right), & \Gamma_{-}^{2}=\frac{1}{2}\left(\Gamma^{2}-i \Gamma^{3}\right) .
\end{array}
$$

Explicitly

$$
\begin{aligned}
\Gamma_{+}^{1}=\left(\begin{array}{cccc}
0 & 0 & -i & 0 \\
0 & 0 & 0 & i \\
0 & 0 & 0 & 0 \\
0 & 0 & 0 & 0
\end{array}\right), & \Gamma_{-}^{1}=\left(\begin{array}{cccc}
0 & 0 & 0 & 0 \\
0 & 0 & 0 & 0 \\
i & 0 & 0 & 0 \\
0 & -i & 0 & 0
\end{array}\right), \\
\Gamma_{+}^{2}=\left(\begin{array}{cccc}
0 & 1 & 0 & 0 \\
0 & 0 & 0 & 0 \\
0 & 0 & 0 & 1 \\
0 & 0 & 0 & 0
\end{array}\right), & \Gamma_{-}^{2}=\left(\begin{array}{cccc}
0 & 0 & 0 & 0 \\
1 & 0 & 0 & 0 \\
0 & 0 & 0 & 0 \\
0 & 0 & 1 & 0
\end{array}\right)
\end{aligned}
$$

The Dirac equation reads

$$
\not D \psi=\sum_{i=0}^{3} \Gamma^{i}\left[X_{i}, \psi\right]=\lambda \psi
$$

or in terms of the $C$ and $S$ operators

$$
\not D \psi=\Gamma_{-}^{1}[C, \psi]+\Gamma_{+}^{1}\left[C^{\dagger}, \psi\right]+\Gamma_{-}^{2}[S, \psi]+\Gamma_{+}^{2}\left[S^{\dagger}, \psi\right]=\lambda \psi .
$$

In matrix form, the Dirac operator becomes

$$
\not D=\left(\begin{array}{cccc}
0 & {\left[S^{\dagger},\right]} & -i\left[C^{\dagger},\right] & 0 \\
{[S,]} & 0 & 0 & i\left[C^{\dagger},\right] \\
i\left[C^{\dagger},\right] & 0 & 0 & {\left[S^{\dagger},\right]} \\
0 & -i[C,] & {[S,]} & 0
\end{array}\right)
$$


As an ansatz for a four component spinor we take

$$
\psi_{n m}=\left(\begin{array}{c}
|n, m-1\rangle a_{n m} \\
|n, m\rangle b_{n m} \\
|n+1, m-1\rangle c_{n m} \\
|n+1, m\rangle d_{n m}
\end{array}\right)
$$

where $a_{n m}, b_{n m}, c_{n m}, d_{n m} \in \mathbb{C}$, and $|n, m\rangle=C^{n} S^{m} \in \mathcal{A}_{N}$. Using the identities

$$
\begin{aligned}
& {[C,|n m\rangle]=\left(1-q^{-m}\right)|n+1, m\rangle, \quad\left[C^{\dagger},|n m\rangle\right]=\left(1-q^{m}\right)|n-1, m\rangle,} \\
& {[S,|n m\rangle]=-\left(1-q^{-n}\right)|n, m+1\rangle, \quad\left[S^{\dagger},|n m\rangle\right]=-\left(1-q^{n}\right)|n, m-1\rangle,}
\end{aligned}
$$

the Dirac equation $\gamma^{i}\left[X_{i}, \psi_{n m}\right]=\lambda_{n m} \psi_{n m}$ becomes explicitly

$$
\left(\begin{array}{cccc}
-\lambda_{n m} & -\left(1-q^{n}\right) & -i\left(1-q^{m-1}\right) & 0 \\
-\left(1-q^{-n}\right) & -\lambda_{n m} & 0 & i\left(1-q^{m}\right) \\
i\left(1-q^{-m+1}\right) & 0 & -\lambda_{n m} & -\left(1-q^{n+1}\right) \\
0 & -i\left(1-q^{-m}\right) & -\left(1-q^{-n-1}\right) & -\lambda_{n m}
\end{array}\right)\left(\begin{array}{c}
|n, m-1\rangle a_{n m} \\
|n, m\rangle b_{n m} \\
|n+1, m-1\rangle c_{n m} \\
|n+1, m\rangle d_{n m}
\end{array}\right)=0 .
$$

Setting the determinant of the matrix to zero gives

$$
\begin{aligned}
0= & \lambda_{n m}^{4}+\lambda_{n m}^{2}\left(-8+q^{1-m}+q^{-1+m}+q^{-m}+q^{m}+q^{-1-n}+q^{-n}+q^{n}+q^{1+n}\right) \\
& +\left(q^{-1 / 2-n}+q^{1 / 2-m}-2 q^{-1 / 2}-2 q^{1 / 2}+q^{-1 / 2+m}+q^{1 / 2+n}\right)^{2} .
\end{aligned}
$$

This can be written in terms of quadratic $q$-numbers

$$
\begin{aligned}
0= & \lambda_{n m}^{4}+c_{N} \lambda_{n m}^{2}\left([1-m]^{2}+[m]^{2}+[1+n]^{2}+[n]^{2}\right) \\
& +c_{N}^{2}\left([1 / 2+n]^{2}-2[1 / 2]^{2}+[1 / 2-m]^{2}\right)^{2} .
\end{aligned}
$$

The factor $c_{N}$ can be absorbed by a rescaling $\lambda_{n m} \rightarrow \sqrt{c_{N}} \lambda_{n m}$, so that

$$
0=\lambda_{n m}^{4}+\lambda_{n m}^{2}\left([1-m]^{2}+[m]^{2}+[1+n]^{2}+[n]^{2}\right)+\left([1 / 2+n]^{2}-2[1 / 2]^{2}+[1 / 2-m]^{2}\right)^{2} .
$$

This has four solutions, given by

$$
\begin{aligned}
\lambda_{n m ; 1,2,3,4}= & \pm\left\{-\left([1-m]^{2}+[m]^{2}+[1+n]^{2}+[n]^{2}\right)\right. \\
& \pm\left(\left([1-m]^{2}+[m]^{2}+[1+n]^{2}+[n]^{2}\right)^{2}\right. \\
& \left.\left.-\left([1 / 2-m]^{2}+[1 / 2+n]^{2}-2[1 / 2]^{2}\right)^{2}\right)^{1 / 2}\right\}^{1 / 2} .
\end{aligned}
$$

For the modes $n, m=0$, the eigenvalues are $\lambda_{00 ; 1,2}=0$ and $\lambda_{00 ; 3,4}= \pm \sqrt{2}$. In the semiclassical limit, these eigenvalues reduce to

$$
\lambda_{n m ; 1,2,3,4}= \pm\left\{-\left(-1+m-m^{2}-n-n^{2}\right) \pm\left(1-2 m+2 m^{2}+2 n+2 n^{2}\right)^{1 / 2}\right\}^{1 / 2} .
$$

Note that this does not and should not agree with the spectrum of the Dirac operator on a noncommutative torus $T_{\theta}^{2}$ in the sense of $[7,15]$ with infinite-dimensional algebra $\mathcal{A}$, since the differential calculus here is based on inner derivations, while for $T_{\theta}^{2}$ it is based on exterior derivations. 


\section{Conclusion}

We studied general fuzzy tori with algebra of functions $\mathcal{A}=M_{N}(\mathbb{C})$ as realized in Yang-Mills matrix models, and discussed in detail their effective geometry. Our main result is that if certain divisibility conditions are satisfied, then the tori can have non-trivial effective geometry. The corresponding modular space of such fuzzy tori is studied, and characterized in terms of a "fuzzy" modular group $\operatorname{PSL}\left(2, \mathbb{Z}_{N}\right)$. We determined the irreducible spectrum of the Laplace operator on these tori, and exhibit their invariance under $\operatorname{PSL}\left(2, \mathbb{Z}_{N}\right)$. In the semiclassical limit, the general commutative torus represented by two generic vectors in the complex plane is recovered, with generic modular parameter $\tau$. This is quite remarkable since the "apparent" embedding is always rectangular.

The results of this paper demonstrate the generality of the class of fuzzy embedded noncommutative spaces with quantized algebra of functions $\mathcal{A}=M_{N}(\mathbb{C})$. Moreover, our results suggest applications of the fuzzy torus to regularize field-theoretical or string-theoretical models involving tori. A more detailed description of the moduli space (10) would be desirable, which requires a detailed understanding of the structure of $\operatorname{PSL}\left(2, \mathbb{Z}_{N}\right)$ for non-prime integers $N$. Our results also suggest the possibility to define fuzzy analogs of modular forms. We leave an exploration of these topics to future work.

\section{Acknowledgments}

This work was supported by the Austrian Science Fund (FWF) under the contracts P21610 and P24713.

\section{References}

[1] Alexanian G., Balachandran A.P., Immirzi G., Ydri B., Fuzzy $\mathbb{C P}^{2}$, J. Geom. Phys. 42 (2002), 28-53, hep-th/0103023.

[2] Arnlind J., Choe J., Hoppe J., Noncommutative minimal surfaces, arXiv:1301.0757.

[3] Arnlind J., Hoppe J., Huisken G., Discrete curvature and the Gauss-Bonnet theorem, arXiv:1001.2223.

[4] Aschieri P., Grammatikopoulos T., Steinacker H., Zoupanos G., Dynamical generation of fuzzy extra dimensions, dimensional reduction and symmetry breaking, J. High Energy Phys. 2006 (2006), no. 9, 026, 26 pages, hep-th/0606021.

[5] Banks T., Fischler W., Shenker S.H., Susskind L., M theory as a matrix model: a conjecture, Phys. Rev. D 55 (1997), 5112-5128, hep-th/9610043.

[6] Chaichian M., Demichev A., Prešnajder P., Sheikh-Jabbari M.M., Tureanu A., Quantum theories on noncommutative spaces with nontrivial topology: Aharonov-Bohm and Casimir effects, Nuclear Phys. B 611 (2001), 383-402, hep-th/0101209.

[7] Connes A., Douglas M.R., Schwarz A., Noncommutative geometry and matrix theory: compactification on tori, J. High Energy Phys. 1998 (1998), no. 2, 003, 35 pages, hep-th/9711162.

[8] Grosse H., Klimčík C., Prešnajder P., On finite 4D quantum field theory in non-commutative geometry, Comm. Math. Phys. 180 (1996), 429-438, hep-th/9602115.

[9] Grosse H., Prešnajder P., The Dirac operator on the fuzzy sphere, Lett. Math. Phys. 33 (1995), $171-181$.

[10] Hofman C., Verlinde E., U-duality of Born-Infeld on the noncommutative two-torus, J. High Energy Phys. 1998 (1998), no. 12, 010, 21 pages, hep-th/9810116.

[11] Hofman C., Verlinde E., Gauge bundles and Born-Infeld on the non-commutative torus, Nuclear Phys. B 547 (1999), 157-178, hep-th/9810219.

[12] Hoppe J., Some classical solutions of membrane matrix model equations, in Strings, Branes and Dualities (Cargèse, 1997), NATO Adv. Sci. Inst. Ser. C Math. Phys. Sci., Vol. 520, Kluwer Acad. Publ., Dordrecht, 1999, 423-427, hep-th/9702169.

[13] Ishibashi N., Kawai H., Kitazawa Y., Tsuchiya A., A large-N reduced model as superstring, Nuclear Phys. B 498 (1997), 467-491, hep-th/9612115. 
[14] Kimura Y., Noncommutative gauge theories on fuzzy sphere and fuzzy torus from matrix model, Progr. Theoret. Phys. 106 (2001), 445-469, hep-th/0103192.

[15] Landi G., Lizzi F., Szabo R.J., From large $N$ matrices to the noncommutative torus, Comm. Math. Phys. 217 (2001), 181-201, hep-th/9912130.

[16] Madore J., The fuzzy sphere, Classical Quantum Gravity 9 (1992), 69-87.

[17] Nakahara M., Geometry, topology and physics, 2nd ed., Graduate Student Series in Physics, Institute of Physics, Bristol, 2003.

[18] Steinacker H., Emergent gravity and noncommutative branes from Yang-Mills matrix models, Nuclear Phys. B $\mathbf{8 1 0}$ (2009), 1-39, arXiv:0806.2032.

[19] Steinacker H., Emergent geometry and gravity from matrix models: an introduction, Classical Quantum Gravity 27 (2010), 133001, 46 pages, arXiv:1003.4134.

[20] Steinacker H., Non-commutative geometry and matrix models, PoS Proc. Sci. (2011), PoS(QGQGS2011), 004, 27 pages, arXiv:1109.5521. 